\title{
Validación del sistema comprehensivo de Exner en el test de Zulliger
}

\author{
Sylvia Rivera Carpio \& Milagros Cubas Lugón \\ Universidad de Lima
}

Recibido: 12 de abril del 2011 / Aprobado: 16 de junio del 2011

Se trata de una investigación psicométrica que busca validar el sistema comprehensivo de Exner (SC) desarrollado para el psicodiagnóstico de Rorschach al test de Zulliger. El SC permite una evaluación completa de la personalidad, asegurando los criterios psicométricos de validez y confiabilidad. Con este objetivo, se administró el test de Zulliger en conjunto con el de Rorschach y una ficha sociodemográfica, en una muestra de 82 trabajadores entre los 19 y 40 años. Se analizaron los datos empleando entre otras medidas de distribución central como el tamaño de la diferencia de Cohen, correlaciones de Spearman y análisis factorial. Se consideraron únicamente niveles de significación de 0.05 y 0.01.

Test de Zulliger / psicodiagnóstico de Rorschach / Sistema comprehensivo de Exner

Validation of Exner's comprehensive system of Zulliger test

The goal of this psychometric investigation is the validation the Exner's Comprehensive System (CS) developed for the Rorschach psychodiagnosis to be used by the Zulliger Test. CS sustains a complete evaluation of personality with validity and reliability. In this study the Zulliger Test was administered together with the Rorschach Test and a socio-demographic file with a sample of 82 workers ages 19-40. Data were analyzed using measurements of central distribution such as Cohen's d, Spearman's correlation rank and factorial analysis. The level of significance was placed between 0.05 and 0.01 .

\section{Zulliger Test / Rorschach psychodiagnosis / Exner's comprehensive system}

Correos electrónicos: srivera@ulima.edu.pe mcubas@ulima.edu.pe 


\section{INTRODUCCIÓN}

En la actualidad, dentro de la batería de pruebas para la selección de personal, el test de Zulliger sobresale como uno de los más rigurosos y completos en la evaluación de la personalidad. Este instrumento se sustenta en los mismos principios que el psicodiagnóstico de Rorschach al emplear como estímulos manchas de tinta. No obstante, dado que solo consta de tres láminas, su administración y codificación demanda menos tiempo. Por ello, a nivel internacional es considerado como el instrumento más eficiente, confiable y práctico para la tarea de selección de personal.

El test de Zulliger fue creado en 1942 por Hans Zulliger, a partir de la necesidad de una prueba colectiva completa de evaluación de personalidad. Posteriormente, en 1954 se publica la versión individual de "Der Tafeln Z Test" ("El test $\mathrm{Z}$ de Láminas"). Si bien este test fue utilizado originalmente como una prueba proyectiva, en los últimos años se le ha extrapolado de manera intuitiva al sistema comprehensivo de Exner (1994) creado para el psicodiagnóstico de Rorschach. La razón de esto reside en que el SC ha mostrado en el Rorschach validez y confiabilidad en la evaluación completa de la personalidad en las siguientes áreas: afecto, capacidad de control y tolerancia al estrés, procesamiento de la información, relaciones interpersonales y autopercepción.
El sistema comprehensivo de Exner toma la propuesta de Rorschach y concibe la prueba como una tarea cognitivo-perceptual en la que los estímulos presentados son organizados por el evaluado a partir de sus características cognitivas y de personalidad. Desde 1968, la Rorschach Research Foundation, promotora de este sistema, se avocó a la integración de las diferentes modalidades de codificar e interpretar la prueba del Rorschach en un lenguaje común, con la finalidad de incorporarla a las ventajas de la psicometría. Como resultado, se ha probado en múltiples investigaciones su validez y confiabilidad y es considerada actualmente como un instrumento científico con valor psicométrico probado.

En 1990, dadas las similitudes existentes entre el psicodiagnóstico de Rorschach y el test de Zulliger, el Grupo de Interés por el Zulliger (ZIG, en Mattlar et al., 1990) en Finlandia, hizo una adaptación del sistema comprehensivo (SC) desarrollado por Exner para el Rorschach al test de Zulliger, con el fin de acercarlo a las ventajas de la psicometría; lo que fue replicado posteriormente en Latinoamérica por Zdunic (2008). No obstante, no se encontraron estudios previos de validez del SC en el test de Zulliger, con lo que las adaptaciones referidas estarían asumiendo dicha validez.

En el Perú, el test de Zulliger empieza a ser introducido por grandes con- 
sultoras, al ser considerada en Latinoamérica como la prueba más eficiente y pertinente para una evaluación completa tanto de la personalidad como de los recursos y habilidades. No obstante, su difusión se ve restringida por la ausencia de normas acordes a nuestra realidad, lo que dificulta el establecimiento de un perfil psicológico válido y confiable y la contrastación de los resultados obtenidos con un grupo de pertenencia.

\section{Metodología}

Por los motivos expuestos, en la presente investigación se propuso:

- Validar el sistema comprehensivo desarrollado por Exner para el psicodiagnóstico de Rorschach, en el test de Zulliger, a partir de un grupo de profesionales en Lima metropolitana.

- Determinar la validez de constructo y concurrente del sistema comprehensivo desarrollado por Exner para el psicodiagnóstico de Rorschach, en el test de Zulliger.

- Describir las propiedades psicométricas del test de Zulliger según el $\mathrm{SC}$ de Exner.

Para ello, se realizó un estudio psicométrico no experimental, descriptivo y de corte transversal (Hernández, Fernández \& Baptista, 2010). Se utilizó un muestreo no probabilístico intencional. La muestra estuvo compuesta por 82 profesionales laboralmente activos en Lima, de ambos géneros, entre los 19 y 40 años y sin antecedentes de patología mental considerable o severa.

\section{Procedimiento}

Se utilizó el procedimiento de administración desarrollado por el sistema comprehensivo de Exner en el caso del psicodiagnóstico de Rorschach, y las recomendaciones realizadas por Zdunik (2008) para el test de Zulliger. Los datos descriptivos fueron comparados con las tablas normativas de Zdunik (2008) y de Villemor-Amaral (2009), en el caso del test de Zulliger; y con las normas de Lima metropolitana (Ráez, 2007) para el Rorschach. Se procedió a realizar las aplicaciones de ambas pruebas, evaluándose en la primera mitad de la muestra con el psicodiagnóstico de Rorschach, mientras que en la segunda mitad se realizó una primera aplicación del test de Zulliger. Quince días después de la primera se realizó la aplicación de la segunda prueba, para así evitar el efecto de la influencia de una prueba en la segunda. Posteriormente, decodificados los protocolos, se realizó la confiabilidad entre evaluadores, obteniéndose valores que oscilan desde $91,7 \%$ hasta $100 \%$, reflejando estos un acuerdo sustancial.

Establecida la confiabilidad, se procedió a realizar el estudio de validez, utilizándose para ello el método de triangulación al contrastar los datos aportados por los instrumentos. Una 
vez obtenida la base de datos completa se calcularon los estadísticos descriptivos de ambas pruebas para luego prorratear dichos valores -considerando que el Rorschach presenta una mayor cantidad de estímulos, lo que aumentaría la frecuencia de sus respectivas variables con respecto al Zulliger-, y comparar las medidas de tendencia central y las medidas de las distribuciones en ambas pruebas. Posteriormente, se buscaron correlaciones altas entre los indicadores del Rorschach y del Zulliger, empleándose con este fin la correlación de Spearman, al tratarse de estadísticos no paramétricos. Únicamente se incluyeron las variables con valores $\mathrm{p}$ $<0.05^{*}$ y $\mathrm{p}<0.01 * *$, al ser consideradas significativas. El programa estadístico utilizado para analizar los datos fue el SPSS 17.

Al resultar significativa la correlación entre el número de respuestas en ambas pruebas, se construyó un modelo de regresión únicamente en el caso de la variable de número de respuestas (R), para así discriminar el número mínimo requerido para que el protocolo de Zullinger sea válido. Adicionalmente, se establecieron las características normativas del test de Zulliger para el grupo de participantes, así como sus estilos vivenciales. Para ello, como referencia se utilizó la diferencia entre los dos lados de la EB, criterio empleado por Exner para el Rorschach, y asumido por Zdunik para el Zulliger. Por último, con la finalidad de obtener validez de constructo entre las variables, se realizó un análisis factorial agrupando las 73 variables del estudio en función de las siete agrupaciones que han sido descritas para el SC de Exner. Con este fin, se empleó el análisis factorial oblimin para factores correlacionados.

\section{Resultados}

Se hallaron indicadores de validez concurrente en una serie de variables, que representan los aspectos centrales del sistema comprehensivo de Exner a partir de la teoría generada en el psicodiagnóstico de Rorschach. Entre ellas se encuentran el número de respuestas (R), respuestas de localización de detalle usual (D), calidad evolutiva ordinaria (DQo), determinante de movimiento humano (M), determinante de forma pura (F), respuestas de par (2), respuestas populares $(\mathrm{P})$; los contenidos humanos $(\mathrm{H})$, animal (A), detalle animal $(\mathrm{Ad})$, botánica $(\mathrm{Bt})$, sexo $(\mathrm{Sx})$ e idiográfico (Id); y finalmente los códigos especiales de verbalización desviada de nivel 1 (DV), contenido de movimiento cooperativo (COP) y buenas representaciones humanas (GHR). La existencia de esta proporcionalidad le da sustento a la validez del uso del sistema comprehensivo de Exner creado para el Rorschach en el test de Zulliger.

Adicionalmente, se encontraron numerosas variables que muestran similitud en ambas pruebas en la medida de tendencia central, así como en sus dis- 
tribuciones, lo que permite anticipar el comportamiento de las variables de localización global (W), calidad formal única (FQxu) y calidad formal distorsionada (FQx-), algunos determinantes, índice de intelectualización (Intelect), estimulación sufrida (es), respuestas complejas (compl.), algunos contenidos y ciertos códigos especiales. Todo ello nos permitirá afirmar que existe cierta semejanza entre las variables del Psicodiagnóstico de Rorschach y el Test de Zulliger, sin que esto implique equivalencia entre ambas pruebas. A continuación, se detallará lo observado en las variables anteriormente mencionadas.

Por ejemplo, en la variable de localización de respuesta global (W) la calidad formal única (FQxu) y distorsionada (FQx-) se hallaron distribuciones similares así como medidas de tendencia central similares. Dentro de los determinantes, se encontraron valores similares en el movimiento animal (FM), color forma (CF), tipos de respuesta de color acromático (FC', C'F y C'), forma textura (FT), vista forma (VF), sombreado forma (YF), sombreado puro (Y), suma de color acromático (Sum C'), suma de textura (Sum T), suma de vista (Sum V), y en las proporciones del determinante de movimiento humano pasivo (Mp). A nivel de las variables de control y tolerancia al estrés se obtuvieron similitudes en la variable de estimulación sufrida (es), en el índice de intelectualización (Intelect.) y en las respuestas complejas (Compl.). En las variables de los contenidos se observaron valores similares en los tipos de contenidos humanos (exceptuando el contenido de $\mathrm{H}$ pura), así como en los contenidos anatómico (An), fuego (Fi), comida (Fd), geografìa (Ge), y naturaleza $(\mathrm{Na})$. Con respecto a los códigos especiales, se encontraron similitudes en los códigos críticos de: respuesta desviada (DR), combinación fabulatoria de nivel 1 (Fabcom 1), verbalización desviada de nivel 2 (DV2) y lógica inadecuada (Alog). También se hallaron similitudes en códigos especiales de contenido de representaciones humanas pobres (PHR), contenido mórbido (MOR), respuesta personalizada (PER) y perseveración (PSV).

Por otro lado, se encontró algunas variables que presentan correlaciones medianas y altas, lo cual implica que existe proporcionalidad entre ambas pruebas. Tal es el caso del número de respuestas $\mathrm{R}$, respuestas de localización de detalle usual (D), calidad evolutiva ordinaria (DQo), determinante de movimiento humano $(\mathrm{M})$, determinante de forma pura (F), respuestas de par (2), respuestas populares $(\mathrm{P})$, los contenidos humanos (H), animal (A), detalle animal (Ad), botánica (Bt), sexo (Sx) e idiográfico (Id); y finalmente los códigos especiales de verbalización desviada de nivel 1 (DV), contenido de movimiento cooperativo (COP) y buenas representaciones humanas (GHR) (véase la tabla 1). 
Tabla 1

Comparación de tendencia central, de distribuciones y de correlaciones medianas y altas

\begin{tabular}{lccccc}
\hline & \multicolumn{2}{c}{ P. de Wilcoxon } & P. de signos & \multicolumn{2}{c}{ Correlaciones } \\
\hline Códigos & Sig. & d de Cohen & Sig. & R & Sig. \\
\hline R & 0,00 & 0,95 & 0,00 & 0,59 & 0,00 \\
D & 0,00 & 0,77 & 0,00 & 0,36 & 0,00 \\
DQ o & 0,00 & 0,61 & 0,00 & 0,53 & 0,00 \\
M & 0,00 & 0,6 & 0,00 & 0,51 & 0,00 \\
FM & 0,06 & 0,14 & 0,05 & 0,50 & 0,00 \\
F & 0,00 & 0,68 & 0,00 & 0,48 & 0,00 \\
Par & 0,00 & 0,54 & 0,00 & 0,52 & 0,00 \\
Populares & 0,00 & 1,06 & 0,00 & 0,31 & 0,00 \\
H & 0,00 & 0,97 & 0,00 & 0,42 & 0,00 \\
A & 0,00 & 0,66 & 0,00 & 0,45 & 0,00 \\
(Ad) & 0,00 & 0,75 & 0,00 &. & 0,00 \\
Bt & 0,00 & 0,85 & 0,01 & 0,35 & 0,00 \\
Sx & 0,06 & 0,57 & 0,06 & $\cdot$ & 0,00 \\
Idio & 0,00 & 0,55 & 0,02 & 0,33 & 0,00 \\
DV & 0,08 & 0,24 & 0,61 & 0,33 & 0,00 \\
COP & 0,00 & 0,63 & 0,02 & 0,42 & 0,00 \\
GHR & 0,00 & 0,63 & 0,00 & 0,44 & 0,00 \\
\hline
\end{tabular}

Tabla 2

Comparación de tendencia central, de distribuciones y de correlaciones bajas

\begin{tabular}{lccccc}
\hline & \multicolumn{2}{c}{ P. de Wilcoxon } & P. de signos & \multicolumn{2}{c}{ Correlaciones } \\
\hline Códigos & Sig. & d de Cohen & Sig. & R & Sig. \\
\hline S & 0,02 & 0,46 & 0,09 & 0,18 & 0,07 \\
FQXo & 0,00 & 1,05 & 0,00 & 0,23 & 0,03 \\
FQX- & 0,09 & 0,15 & 0,08 & 0,21 & 0,03 \\
FC & 0,00 & 0,68 & 0,04 & 0,27 & 0,01 \\
CF & 0,21 & 0,19 & 1,00 & 0,28 & 0,01 \\
FC' & 0,64 & 0,12 & 0,14 & 0,19 & 0,05 \\
p (pasivo) & 0,10 & 0,22 & 0,02 & 0,24 & 0,02 \\
Ad & 0,11 & 0,15 & 0,00 & 0,23 & 0,02 \\
An & 0,52 & 0,18 & 0,20 & 0,29 & 0,01 \\
Art & 0,08 & 0,11 & 0,00 & 0,19 & 0,05 \\
Ay & 0,31 & 0,23 & 0,22 & 0,22 & 0,03 \\
Fi & 0,90 & 0,08 & 0,2 & 0,29 & 0,01 \\
Ge & 0,34 & 0,1 & 0,07 & 0,18 & 0,06 \\
Hh & 0,82 & 0,13 & 0,02 & 0,22 & 0,03 \\
Na & 0,75 & 0,04 & 0,4 & 0,27 & 0,01 \\
AG & 0,00 & 0,44 & 0,12 & 0,24 & 0,02 \\
PER & 0,90 & 0,02 & 0,21 & 0,25 & 0,01 \\
\hline
\end{tabular}


Tal como se presenta en la tabla 2 , se halló una serie de variables que muestran similitud en la medida de tendencia central así como en sus distribuciones, sin embargo, presentan solo correlaciones bajas entre ambas pruebas. Esto significa que la coincidencia entre dichas variables no implica una correspondencia entre ellas, y por tanto no se puede predecir el comportamiento de los mismos en aplicaciones futuras. Entre estos códigos se encuentran: las respuestas de espacio en blanco (S), la calidad formal distorsionada (FQ-), los determinantes de forma color (FC), color forma $(\mathrm{CF})$ y forma color acromático (FC'), el superíndice de movimiento pasivo (p), los contenidos de detalle animal (Ad), anatomía (An), arte (Art), antropología (Ay), fuego (Fi), geografía
(Ge), hogar (Hh) y naturaleza (Na); y el código especial de contenido de personalización (PER). Adicionalmente, se ha encontrado que la variable de calidad formal ordinaria (FQo) presenta distribuciones disímiles entre las pruebas y una correlación baja. En este caso en particular, dicha variable requeriría una mayor investigación en el test de Zulliger.

Un aporte esencial de la presente investigación consiste en la designación de un número de respuestas mínimo en el Zulliger para que el protocolo sea considerado válido. En la tabla 3 se observa que el mínimo de respuestas requerido en el test de Zulliger es de 7, encontrándose dicho valor en un intervalo de confianza al 95\% entre el 6.7 y 8.1. Este hallazgo resulta fundamental,

Tabla 3

Análisis de regresión en el cálculo del número mínimo de respuestas en el Zulliger

\begin{tabular}{cccccc}
\hline Modelo & \multicolumn{2}{c}{ Coeficientes no estandarizados } & \multicolumn{3}{c}{ Coeficientes estandarizados } \\
\hline & B & Error típ. & Beta & t & sig \\
\hline 1 (Constante) & 4,92 & 0,73 & & 6,75 & 0,00 \\
ROR_R & 0,17 & 0,03 & 0,57 & 5,93 & 0,00 \\
\hline
\end{tabular}

a Variable dependiente: ZUL_R

Pronóstico

ZUL_r $=4,92+0,17^{*} R O R \_R$

\begin{tabular}{cccc}
\hline R RORSCHACH & Rzulliger & \multicolumn{2}{l}{ Intervalo de confianza 95\% } \\
\hline 14 & 7.4 & 6.7 & 8.1 \\
\hline
\end{tabular}


ya que permite construir normas y baremos válidos dentro de una población.

El test de Zulliger es una prueba que se emplea en selección de personal, por lo que es fundamental registrar los estilos vivenciales, pues en muchos sentidos constituyen el eje central a través del cual va a organizarse la personalidad. Los estilos vivenciales brindan información acerca del estilo de personalidad y de las estrategias usuales de trabajo del evaluado. Por ello, ayudan a comprender la manera en que el evaluado maneja la información del entorno y cómo esta determina su proceso de toma de decisiones y su posible éxito o fracaso.

Otro aporte del presente estudio se ve representado por la determinación de los estilos vivenciales de manera similar a lo propuesto por Exner para el psicodiagnóstico de Rorschach. Tal como se observa en la tabla 4, existe una coincidencia de $25 \%$ en el estilo introversivo entre el test de Zulliger y el psicodiagnóstico de Rorschach, así como en el estilo vivencial extratensivo. Por otro lado, el 16,7\% de la muestra presenta en ambas pruebas una coincidencia en el estilo vivencial ambitendente. Finalmente, se obtuvo en gran proporción de la muestra, con $75,6 \%$, una coincidencia en el estilo evitativo tanto en el Psicodiagnóstico de Rorschach como en el test de Zulliger.

En esta línea, se halló una correlación mediana (0.30) entre los estilos vivenciales propuestos por el SC de Ex-

Tabla 4

Distribución de la muestra de acuerdo con los estilos vivenciales

\begin{tabular}{llcccccccccc}
\hline & \multicolumn{10}{c}{ EstVivROR } \\
\cline { 3 - 13 } & & \multicolumn{1}{c}{ Introversivo } & Ambitendente & Extratensivo & \multicolumn{1}{c}{ Evitativo } & \multicolumn{2}{c}{ Total } \\
\cline { 3 - 13 } & & $\mathbf{n}$ & $\%$ & $\mathbf{n}$ & $\%$ & $\mathbf{n}$ & $\%$ & $\mathbf{n}$ & $\%$ & $\mathbf{n}$ & $\%$ \\
\hline EstVivZUL & Intriversivo & 3 & 25,0 & 7 & 38,9 & 1 & 25,0 & 5 & 12,2 & 16 & 21,3 \\
Zdunik & Ambitendente & 1 & 8,3 & 3 & 16,7 & 0 & 0,0 & 0 & 0,0 & 4 & 5,3 \\
& Extratensivo & 0 & 0,0 & 3 & 16,7 & 1 & 25,0 & 5 & 12,2 & 9 & 12,0 \\
& Evitativo & 8 & 66,7 & 5 & 27,8 & 2 & 50,0 & 31 & 75,6 & 46 & 61,3 \\
Total & 12 & 100,0 & 18 & 100,0 & 4 & 100,0 & 41 & 100,0 & 75 & 100,0 \\
\hline
\end{tabular}


ner para el Rorschach, y aquellos que Zdunik plantea respectivamente para el Z-test. Ahora, si bien dichos estilos guardan proporcionalidad con los lineamientos ampliamente probados del SC de Exner, se afirma que los valores determinados en el Z-test no pueden ser extrapolados automáticamente en base a las diferencias propias del EB que el SC propone para el test de Rorschach. Por ello, se requiere plantear una nueva proporcionalidad para la determinación de los estilos vivenciales en el test de Zulliger. Esta información resulta importante para la descripción de las estrategias de trabajo de los evaluados, favoreciendo a los candidatos con un reconocimiento más ajustado de sus recursos y estilos.

Se han realizado aproximaciones para la determinación de los estilos vivenciales en el test de Zulliger, lo que aún requiere una profunda investigación que aportará al estudio del estilo de trabajo en los evaluados. Los análisis realizados en este nivel en el presente estudio resultan aún preliminares dado el tamaño de muestra en el mismo.

En la tabla 5 se presenta la distribución de los estilos vivenciales siguiendo los planteamientos realizados por Exner. Luego de realizar una investigación exhaustiva, dicho autor estableció ciertas reglas con respecto a los valores en las variables implicadas para la determinación de los estilos referidos. En este sentido, utiliza la variable de EA (experiencia accesible) como criterio para la definición de dichos estilos de trabajo. Dicha variable se encuentra conformada por la relación entre las variables de movimiento humano (M) y suma ponderada de color (SumPondC). Exner plantea que la diferencia entre las variables de movimiento humano (M) y suma ponderada de color (SumPondC) debe de ser como mínimo de 2 o más puntos para hablar de un estilo viven-

Tabla 5

Distribución de los estilos vivenciales en el Zulliger a partir de Exner

\begin{tabular}{llcccccccccc}
\hline & \multicolumn{1}{c}{ EstVivROR } \\
\cline { 3 - 12 } & & \multicolumn{1}{c}{ Introversivo } & Ambitendente & Extratensivo & \multicolumn{1}{c}{ Evitativo } & \multicolumn{2}{c}{ Total } \\
\cline { 3 - 12 } & & $\mathbf{n}$ & $\%$ & $\mathbf{n}$ & $\%$ & $\mathbf{n}$ & $\mathbf{\%}$ & $\mathbf{n}$ & $\%$ & $\mathbf{n}$ & $\%$ \\
\hline EstVivZUL M- & Intriversivo & 1 & $\mathbf{8 , 3}$ & 3 & 16,7 & 0 & 0,0 & 1 & 2,4 & 5 & 6,7 \\
SumPondC 2 & Ambitendente & 3 & 25,0 & 8 & $\mathbf{4 4 , 4}$ & 2 & 50,0 & 8 & 19,5 & 21 & 28,0 \\
& Extratensivo & 0 & 0,0 & 2 & 11,1 & 0 & $\mathbf{0 , 0}$ & 1 & 2,4 & 3 & 4,0 \\
& Evitativo & 8 & 66,7 & 5 & 27,8 & 2 & 50,0 & 31 & $\mathbf{7 5 , 6}$ & 46 & 61,3 \\
Total & 12 & 100,0 & 18 & 100,0 & 4 & 100,0 & 41 & 100,0 & 75 & 100,0 \\
\hline
\end{tabular}


cial estable, lo que representa a los estilos introversivo y evitativo.

En la tabla 5 se observa que al plantear en el Zulliger un valor mínimo en la diferencia de ambos lados del EB, la coincidencia entre ambas pruebas en particular con respecto al estilo vivencial ambitendente mejora. Sin embargo, disminuye el tamaño de coincidencias con respecto a los estilos vivenciales introversivo y extratensivo. Esto se encontraría a su vez influenciado por el tamaño reducido en la muestra de ambos estilos. Así, únicamente se cuenta con un grupo de 8 participantes que aparecen como introversivos en el test de Rorschach y 4 que figuran como extratensivos.

Se considera que si bien los estilos vivenciales del SC resultan de utilidad en el Test de Zulliger, este último instrumento cuenta con una lógica propia al momento de determinarlos. Por ello, se requiere una mayor investigación al respecto, ya que permitiría una decisión más informada y con mayores herramientas acerca del estilo de trabajo y aspectos que influyen en la motivación y producción del evaluado dentro del ámbito organizacional.
Adicionalmente, se realizó un análisis factorial con la finalidad de establecer si el test de Zulliger en efecto mide los factores postulados por el SC. En la tabla 6 , se consignan los siete componentes y la varianza correspondiente en cada uno de sus casos, los cuales explican el $51 \%$ de la varianza total. Posteriormente, en la tabla 7 , se presenta la matriz de cargas factoriales de análisis de las respuestas, en donde se señalan los componentes de cada uno de los factores hallados.

El factor 1 está compuesto por variables que representan un procesamiento cognitivo elaborado, sofisticado y organizado, a través del cual el evaluado utiliza sus recursos cognitivos disponibles, evidenciando motivación y esfuerzo en dicho proceso. A su vez, es capaz de tomar decisiones de manera activa, pudiendo considerar sus necesidades e intereses y logrando establecer relaciones positivas y saludables con los demás.

El segundo factor implica un enfoque de la realidad poco convencional, a través del cual al evaluado se le dificultaría interpretar las situaciones de ma-

Tabla 6

Varianza de análisis factorial

\begin{tabular}{lccccccc}
\hline Componente & $\mathbf{1}$ & $\mathbf{2}$ & $\mathbf{3}$ & $\mathbf{4}$ & $\mathbf{5}$ & $\mathbf{6}$ & $\mathbf{7}$ \\
Varianza & 10.01 & 4.98 & 5.27 & 6.00 & 4.52 & 5.55 & 3.66 \\
\% de varianza (de 51\%) & 13.71 & 6.82 & 7.22 & 8.22 & 6.19 & 7.60 & 5.02 \\
\hline
\end{tabular}


Tabla 7

Matriz de cargas factoriales de análisis de respuestas

\begin{tabular}{|c|c|c|c|c|c|c|c|}
\hline & & & Cargas & oriales & limin & & \\
\hline & F1 & F2 & F3 & F4 & F5 & F6 & F7 \\
\hline EA & 0.70 & & -0.39 & & & & \\
\hline M & 0.87 & & & & & & \\
\hline COP & 0.44 & & & 0.23 & & 0.28 & \\
\hline GHR & 0.74 & & & 0.19 & & & \\
\hline Todo $\mathrm{H}$ & 0.79 & & & & & & \\
\hline & 0.87 & & & & & & \\
\hline Ls & 0.43 & & & & & & \\
\hline Ma & 0.64 & & & & & 0.23 & \\
\hline $\mathrm{Mp}$ & 0.72 & & & & & & \\
\hline MQ- & 0.56 & 0.35 & & & & & \\
\hline Populares & 0.37 & -0.38 & -0.29 & & & & \\
\hline $\mathrm{Zf}$ & 0.54 & & -0.18 & & & & \\
\hline W & 0.23 & & -0.27 & & & & \\
\hline $\mathrm{DQ}+$ & 0.62 & & & 0.20 & & 0.29 & \\
\hline $3 r+(2) / r$ & 0.44 & -0.41 & & & & & \\
\hline $\mathrm{Fr}+\mathrm{rF}$ & 0.27 & & & & & & \\
\hline $\mathrm{s}$ & & 0.45 & & & & & \\
\hline PHR & 0.30 & 0.67 & & & & & \\
\hline$X A \%$ & & -0.76 & & & & & \\
\hline$X-\%$ & & 0.77 & & & & & \\
\hline S- & & 0.47 & & & & & \\
\hline$x+\%$ & & 0.67 & & & & & -0.28 \\
\hline $\mathrm{Dd}$ & & 0.57 & & & & & 0.28 \\
\hline $\mathrm{Hd}$ & & 0.67 & & & & & \\
\hline $\mathrm{R}$ & & 0.32 & -0.49 & & & & \\
\hline C & & & -0.83 & & & & \\
\hline Sum Pond C & & & -0.58 & 0.25 & -0.48 & & \\
\hline $\mathrm{CP}$ & & & -0.48 & & & 0.35 & \\
\hline $\mathrm{Bt}$ & & & -0.48 & & & -0.31 & \\
\hline $\mathrm{DQ} v$ & & & -0.63 & & & & \\
\hline $\mathrm{Xy}$ & & & -0.38 & & & & \\
\hline Sum C' & & & & 0.74 & & & \\
\hline Sum V & & & & 0.61 & & & \\
\hline Sum Sh & & & & 0.63 & & & 0.35 \\
\hline $\mathrm{FC}$ & & & & 0.64 & & & \\
\hline Compl./R & & & & 0.48 & -0.56 & 0.23 & \\
\hline Sum6 CE2 & & & & 0.59 & & & \\
\hline Sum Pond6 & & & & 0.52 & & & \\
\hline$(\mathrm{H})$ & & & & 0.36 & 0.33 & & \\
\hline $\mathrm{m}$ & & & & & -0.57 & 0.33 & \\
\hline $\mathrm{CF}$ & & & & & -0.76 & & \\
\hline $\mathrm{Zf}$ & & & & & -0.31 & & \\
\hline W & & & & & -0.59 & & \\
\hline D & & & & & 0.58 & & \\
\hline $\mathrm{Dd}$ & & & & & 0.36 & & \\
\hline Lambda & & & & -0.31 & & -0.43 & \\
\hline es & & & & & & 0.74 & \\
\hline FM & & & & & & 0.88 & \\
\hline $\mathrm{FM}+\mathrm{m}$ & & & & & & 0.94 & \\
\hline$A G$ & & & & & & 0.64 & \\
\hline a (activo) & 0.52 & & & & & 0.56 & \\
\hline $\mathrm{p}$ (pasivo) & & & & & & 0.44 & \\
\hline Art & & & & & & 0.26 & \\
\hline Sum $T$ & & & & & & & 0.71 \\
\hline Ay & & & -0.31 & & & & 0.38 \\
\hline MOR & & & & & & & 0.44 \\
\hline PER & & & & & & & 0.45 \\
\hline Xu\% & & & & & & & 0.57 \\
\hline
\end{tabular}


nera similar a la mayoría de personas. Esto afectaría su adaptación a las normas del grupo de pertenencia, pudiendo a su vez estar influido por interferencias emocionales. Asimismo, esto impactaría negativamente en su propia valoración de sí mismo así como la calidad de sus relaciones interpersonales.

El tercer factor muestra un esfuerzo limitado del evaluado por organizar sus respuestas, así como por lograr un funcionamiento más diferenciado y sofisticado que se acompaña de un escaso involucramiento de aspectos afectivos.

El cuarto factor representa un funcionamiento caracterizado por procesos de análisis y síntesis en los que el evaluado integra los aspectos afectivos, representados por la expresión de afectos positivos, así como por la existencia de afectos disfóricos. Estos últimos generan un aumento del estrés, que incide en la calidad del proceso ideacional del evaluado, ante lo que buscaría relaciones interpersonales cooperativas y saludables con los otros.

El quinto factor se vincula con un acercamiento parcial y particular a la realidad, en el que el esfuerzo y motivación del evaluado resultan reducidos, dejándose de lado aspectos afectivos y de situaciones de presión.

El sexto factor se interpreta como cierta preocupación ideacional que limita la capacidad de control y tolerancia al estrés. Frente a ello, el evaluado emplea su capacidad de autonomía, logrando tomar decisiones de manera independiente. Así mismo, refleja la participación en las situaciones interpersonales, por lo que el sujeto podría relacionarse de manera cooperativa y empática o podría defenderse, anticipando aspectos de dichos intercambios.

Por último, el séptimo factor representa aspectos de respuestas poco convencionales, teñidas de una percepción muy particular acompañada de cierto pesimismo, una autopercepción dañada, así como de afectos disfóricos.

Los componentes del análisis factorial mostraron agrupaciones que resultan coherentes con las expectativas y definiciones teóricas para la interpretación del SC. Los aspectos más sólidos que se han hallado en el presente análisis se centran en un procesamiento cognitivo elaborado y diferenciado, un ajuste mediacional particular y distorsionado, un limitado esfuerzo por integrar información, junto con una escasa conexión con los propios afectos.

Al haberse encontrado factores en el test de Zulliger que son inherentes al sistema comprehensivo para el Rorschach, se puede afirmar que la estructura interna es común para ambos instrumentos, lo cual le otorgaría un valor psicométrico al test de Zulliger, al ser el Rorschach un instrumento científico con valor psicométrico probado.

Si bien la proporcionalidad y similitud en las proporciones y frecuencias en ambas pruebas no implica algún tipo de equivalencia se han hallado semejanzas en los indicadores centrales descritos 
por el SC. En esta línea, Exner (1985) propone que si bien la gran mayoría de las variables del SC deben presentarse en los protocolos, existen ciertos indicadores centrales, cuyo registro resulta mucho más relevante. Entre ellos se encuentran las respuestas de movimiento humano (M) y color cromático (FC y $\mathrm{CF}$ ), así como las interpretaciones globales (W) y de detalle (D). En este sentido, las variables que obtuvieron una alta correlación serían aquellas a considerar como principales en el caso de los protocolos del Zulliger.

\section{CONCLUSIONES}

La existencia de proporcionalidad en las variables descritas en el presente estudio le da sustento a la validez del uso del sistema comprehensivo de Exner creado para el Rorschach en el test de Zulliger, pues estas representan los aspectos centrales del sistema comprehensivo.

Así mismo, al haberse encontrado factores comunes inherentes al test de Zulliger que además han sido descritos a partir del SC se puede afirmar que estructuralmente el psicodiagnóstico de Rorschach y el Z-test cuentan con elementos comunes. La presencia de estos rasgos comunes en la estructura interna de ambos instrumentos le otorgaría un valor psicométrico al test de Zulliger.

Por tanto, las sólidas características psicométricas halladas en el test de Zulliger introducen esta prueba en el grupo de instrumentos psicológicos que han sido probados científicamente, a partir de los estrictos requisitos de la psicometría; sin perder, por ello, todas las ventajas que ofrece la interpretación cualitativa originalmente planteada por Hans Zulliger.

Finalmente, aun cuando se ha establecido la validez del SC para el test de Zulliger, cabe señalar que si bien existe cierta proporcionalidad entre las variables del SC en el test de Zulliger y el psicodiagnóstico de Rorschach, esto no indica algún tipo de equivalencia en las variables de ambas pruebas, sino más bien una correlación positiva y alta que evidenciaría cierta similitud en el comportamiento de dichas variables.

Por ello, a pesar de que ciertas variables descritas por el SC de Exner resultan aplicables al test de Zulliger, es necesario ser cautos con las interpretaciones de estas. Por ejemplo, en este último test algunos signos que evalúan recursos y capacidad de adaptación en los evaluados resultan con puntajes más altos que los observados en el psicodiagnóstico de Rorschach, por lo que los evaluados aparecerían como más capaces, con mayores recursos y con mayor capacidad de adaptación.

Por tanto, la construcción de normas propias del test de Zulliger es indispensable para poder discriminar con solidez los candidatos idóneos para los perfiles requeridos en la selección de personal. Para realizar dicha tarea, se considera prioritario evaluar algunos 
aspectos preliminares, tales como la similitud o diferencia formal entre las áreas empleadas en las manchas del test de Rorschach y Zulliger, la estandarización del procedimiento de administración con sus respectivas fases y consignas, el efecto de una secuencia cromática distinta entre ambas pruebas, la designación de los estilos vivenciales de acuerdo al SC E en el Z-test, entre otros. A modo de conclusión, se puede afirmar que el uso del sistema comprehensivo de Exner en el test de Zulliger consiste en una metodología válida que genera datos sólidos que discriminan con detalle información relevante para la selección de personal. Si bien no es recomendable su uso para diagnósticos diferenciales complejos -necesarios por ejemplo en el ámbito clínico-, al ser su objetivo ceñido, el instrumento provee información rica, compleja y significativa, en poco tiempo. En tal sentido, el test de Zulliger se convierte en una herramienta fundamental en la selección de personal y en cualquier ámbito que necesite una evaluación rápida y circunscrita de la personalidad.

\section{ReFERENCIAS}

Exner, J. E. Jr. (1994). El Rorschach un sistema comprehensivo: Fundamen- tos básicos. Vol. 1. (3. ${ }^{\mathrm{a}}$ ed.). Madrid: Psimática.

Hernández, R.; Fernández, C. \& Baptista, P. (2010). Metodología de la investigación (5. ${ }^{a}$ ed.). México D. F.: México: McGraw-Hill.

Mattlar, C. E.; Sandahl, C.; Lindber, S.; Lehtinen, V.; Carlsson, A.; Vesala, P. \& Mahmood, Z. (1990). Methodological issues associated with the application of the comprehensive system when analyzing the Zulliger, and the structural resemblance between the Zulliger and the Rorschach. British Journal of Projective Psycology, 2, 17-27.

Ráez, M. (2007). "Estudio normativo y multicultural con Rorschach SC en el Perú". XXXI Congreso Interamericano de Psicología. México.

Villemor-Amaral, A. E. \& Primi, R. (2009). Teste de Zuliger no sistema compreensivo- ZSC. Forma individual. São Paulo: Casa do Psicólogo.

Zdunic, A. (2008). El test de Zulliger en la evaluación del personal. Aportes del sistema comprehensivo de Exner. Buenos Aires: Paidós.

Zulliger, H. (1970). El test Z, un test individual y colectivo. Buenos Aires: Kapelusz. 